\title{
Modelling of vibrations of a liquid filled tank
}

\author{
Kazimierz Trębacki, Ph. D. \\ Gdansk University of Technology
}

\begin{abstract}

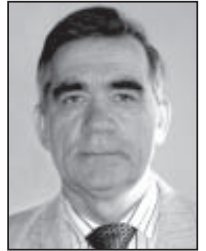

Kinematic excitations provoke the motion and vibrations of the construction, which in turn considerably affects hydrodynamic loads generated on the walls of liquid cargo tanks. The issues of hydrodynamics also refer to the constructions fixed to the bottom, and those half-immersed, fully immersed, and floating (drilling platforms). They mainly concern ship tanks and oceanotechnical tanks. The theoretical and numerical analysis for long an short tanks, fully or partially filled with liquid has been performed. The determined hydrodynamic loads directly refer to the strength of the tank structure, which provides opportunities for determining the constructional strength of the entire hull. Extremely severe requirements are to be met by tanks used for carrying radioactive liquids, as an extremely high safety level is to be secured on all cargo carriers used in marine and land transport.
\end{abstract}

Keywords: modelling, vibrations, hydroelasticity, ship tanks for liquid fuels and liquefied gases

\section{PROBLEM FORMULATION}

A long tank filled with liquid can be modelled by a beam of rectangular cross-section and dimensions $1 \times \mathrm{b}$ x h, with articulated supports at two points. The supports reveal flexibility to vertical movements and rotation $[9 \div 12]$ (see Fig.1).

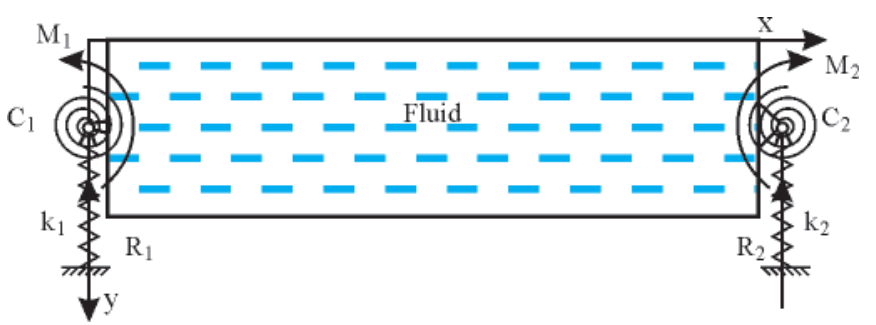

Fig. 1. Scheme of a model liquid-filled tank

where:

$\mathrm{k}_{1}, \mathrm{k}_{2} \quad-$ axial spring constants

$\mathrm{C}_{1}, \mathrm{C}_{2} \quad-$ spiral spring constants

$\mathrm{R}_{1}, \mathrm{R}_{2}, \mathrm{M}_{1}, \mathrm{M}_{2}$ - support reactions and reaction moments, respectively.

When analysing free vibrations of the liquid-filled beam we assume that the functions of beam deflection $\mathrm{w}$ and velocity potential $\varphi$ have the following harmonic form:

$$
\begin{gathered}
\mathrm{w}=\widetilde{\mathrm{w}}(\mathrm{x}) \sin \omega \mathrm{t} \\
\varphi=\tilde{\varphi}(\mathrm{x}, \mathrm{y}, \mathrm{z}) \cos \omega \mathrm{t}
\end{gathered}
$$

The differential equation for the liquid-filled beam is the following [11]:

$$
\begin{gathered}
\mathrm{EI} \frac{\partial^{4} \mathrm{w}}{\partial \mathrm{x}^{4}}-\left(\mathrm{N}_{\mathrm{h}}+\mathrm{N}_{\mathrm{d}}\right) \frac{\partial^{2} \mathrm{w}}{\partial \mathrm{x}^{2}}+ \\
+\rho_{\mathrm{b}} \mathrm{A} \frac{\partial^{2} \mathrm{w}}{\partial \mathrm{t}^{2}}=\mathrm{q}(\mathrm{x})+\int_{0}^{\mathrm{b}}\left(\mathrm{p}_{2}-\mathrm{p}_{1}\right) \mathrm{dz}
\end{gathered}
$$

where:

EI $\quad-$ flexural stiffness

$\rho_{\mathrm{b}} \quad-$ beam material density,

A $\quad-$ cross-section area,

$\mathrm{p}_{2}, \mathrm{p}_{1} \quad-$ wall pressures for $\mathrm{y}=\mathrm{h}$ and $\mathrm{y}=0$,

$\mathrm{N}_{\mathrm{h}}, \mathrm{N}_{\mathrm{d}}$ - longitudinal forces from hydrostatic and hydrodynamic pressure, respectively, acting on top walls for $\mathrm{x}=0$ and $\mathrm{x}=1$.

These forces are determined using the formulas:

$$
\begin{aligned}
& \mathrm{N}_{\mathrm{h}}=\frac{1}{2} \rho g b \mathrm{~h}^{2} \\
& \mathrm{~N}_{\mathrm{d}}=\int_{0}^{\mathrm{h}} \mathrm{p}_{\mathrm{d}} \mathrm{bdy}
\end{aligned}
$$

where:

$\rho \quad-$ liquid density

$\mathrm{g}=-9.81 \mathrm{~ms}^{-2}-$ Earth gravitation. 
The hydrodynamic pressure is calculated from the Bernoulli equation:

$$
p_{d}=-\rho \frac{\partial \varphi}{\partial t}=\rho \omega \tilde{\varphi} \sin \omega t
$$

After putting (6) into relation (5) we get the formula for $\mathrm{N}_{\mathrm{d}}$ :

$$
\mathrm{N}_{\mathrm{d}}=\left.\rho b \omega \sin \omega t \int_{0}^{\mathrm{h}} \widetilde{\varphi}\right|_{x=1} d y
$$

After putting (4) and (5) into the differential equation (3) and, as a substitution for pressures $\mathrm{p}_{2}$ and $\mathrm{p}_{1}$, into the linear part of the Cauchy-Lagrange integral we arrive at:

$\mathrm{EI} \frac{\partial^{4} \mathrm{w}}{\partial \mathrm{x}^{4}}-\left(\frac{1}{2} \rho g b h^{2}-\left.\rho b \int_{0}^{\mathrm{h}} \frac{\partial \varphi}{\partial \mathrm{t}}\right|_{\mathrm{x}=1} \mathrm{dy}\right) \frac{\partial^{2} \mathrm{w}}{\partial \mathrm{x}^{2}}+\rho_{\mathrm{b}} \mathrm{A} \frac{\partial^{2} \mathrm{w}}{\partial \mathrm{t}^{2}}=\mathrm{q}(\mathrm{x})-\rho \mathrm{b}\left(\left.\frac{\partial \varphi}{\partial \mathrm{t}}\right|_{\mathrm{y}=\mathrm{h}}-\left.\frac{\partial \varphi}{\partial \mathrm{t}}\right|_{\mathrm{y}=0}\right)+\rho g b \mathrm{~h}$

This is the non-linear differential equation for transverse vibrations of a long model tank filled with liquid.

For the liquid inside the tank the Laplace equation is solved

$$
\nabla^{2} \varphi=0
$$

with the following boundary conditions for the liquid velocity potential:

$$
\begin{gathered}
\frac{\partial \varphi}{\partial \mathrm{z}}=0 \text { in the } \mathrm{z} \text {-axis direction: } \mathrm{z}=-\frac{\mathrm{b}}{2} \mathrm{i} \mathrm{z}=\frac{\mathrm{b}}{2} \\
\frac{\partial \varphi}{\partial \mathrm{y}}=\frac{\partial \mathrm{w}}{\partial \mathrm{t}} \text { for } \mathrm{y}=0 \text { and } \mathrm{y}=\mathrm{h} \\
\frac{\mathrm{in} \text { the } \mathrm{x} \text {-axis direction: }}{\partial \varphi}=-\left(\mathrm{y}-\frac{\mathrm{h}}{2}\right) \frac{\partial^{2} \mathrm{w}}{\partial \mathrm{x} \partial \mathrm{t}} \text { for } \mathrm{x}=0 \text { and } \mathrm{x}=1
\end{gathered}
$$

After dividing equation (8) by stiffness EI, neglecting the smal non-linear term and assuming that $\mathrm{q}(\mathrm{x})=0$ we arrive at the following new form of the differential equation:

$$
\begin{aligned}
& \frac{\partial^{4} \mathrm{w}}{\partial \mathrm{x}^{4}}-\mathrm{B} \frac{\partial^{2} \mathrm{w}}{\partial \mathrm{x}^{2}}+\mathrm{B}_{1} \frac{\partial^{2} \mathrm{w}}{\partial \mathrm{t}^{2}}=\mathrm{B}_{2}-\mathrm{B}_{3}\left(\left.\frac{\partial \varphi}{\partial \mathrm{t}}\right|_{\mathrm{y}=\mathrm{h}}-\left.\frac{\partial \varphi}{\partial \mathrm{t}}\right|_{\mathrm{y}=0}\right) \\
& \mathrm{B} \equiv \frac{\rho \mathrm{gbh}^{2}}{2 \mathrm{EI}} ; \mathrm{B}_{1} \equiv \frac{\rho_{\mathrm{b}} \mathrm{A}}{\mathrm{EI}} ; \mathrm{B}_{2} \equiv \frac{\rho \mathrm{gbh}}{\mathrm{EI}} ; \mathrm{B}_{3} \equiv \frac{\rho \mathrm{b}}{\mathrm{EI}}
\end{aligned}
$$

This equation includes the deflection function $\mathrm{w}$ and the flow velocity potential $\varphi$. In order to get the partial differential vibration equation which only includes the flow velocity potential, equation (13) is to be differentiated with respect to time t, and then the boundary condition (11) for velocity is to be adopted. Then we get:

$$
\frac{\partial^{5} \varphi}{\partial \mathrm{x}^{4} \partial \mathrm{y}}-\mathrm{B} \frac{\partial^{3} \varphi}{\partial \mathrm{x}^{2} \partial \mathrm{y}}+\mathrm{B}_{1} \frac{\partial^{3} \varphi}{\partial \mathrm{t}^{2} \partial \mathrm{y}}=-\mathrm{B}_{3}\left(\left.\frac{\partial^{2} \varphi}{\partial \mathrm{t}^{2}}\right|_{\mathrm{y}=\mathrm{h}}-\left.\frac{\partial^{2} \varphi}{\partial \mathrm{t}^{2}}\right|_{\mathrm{y}=0}\right) \text { for } \mathrm{y}=0
$$

As we can see, this is the fifth order linear partial differential equation which only includes the velocity potential function $\varphi(\mathrm{x}, \mathrm{y}, \mathrm{z} ; \mathrm{t})$.

The boundary conditions for the supports have the form:

$$
\begin{aligned}
& \left.\frac{\partial^{3} \mathrm{w}}{\partial \mathrm{x}^{3}}\right|_{\mathrm{x}=0}=-\frac{\mathrm{k}_{1}}{\mathrm{EI}} \mathrm{w}(0) ;\left.\frac{\partial^{3} \mathrm{w}}{\partial \mathrm{x}^{3}}\right|_{\mathrm{x}=1}=-\frac{\mathrm{k}_{0}}{\mathrm{EI}} \mathrm{w}(1) \\
& \left.\frac{\partial^{2} \mathrm{w}}{\partial \mathrm{x}^{2}}\right|_{\mathrm{x}=0}=\left.\frac{\mathrm{C}_{1}}{\mathrm{EI}} \frac{\partial \mathrm{w}}{\partial \mathrm{x}}\right|_{\mathrm{x}=0}-\frac{\rho g b h^{3}}{12 \mathrm{EI}}+\left.\frac{\rho \mathrm{b}}{\mathrm{EI}} \int_{0}^{\mathrm{h}} \frac{\partial \varphi}{\partial \mathrm{t}}\right|_{\mathrm{x}=0} \mathrm{ydy} \\
& \left.\frac{\partial^{2} \mathrm{w}}{\partial \mathrm{x}^{2}}\right|_{\mathrm{x}=1}=\left.\frac{\mathrm{C}_{2}}{\mathrm{EI}} \frac{\partial \mathrm{w}}{\partial \mathrm{x}}\right|_{\mathrm{x}=1}-\frac{\rho g b h^{3}}{12 \mathrm{EI}}+\left.\frac{\rho \mathrm{b}}{\mathrm{EI}} \int_{0}^{\mathrm{h}} \frac{\partial \varphi}{\partial \mathrm{t}}\right|_{\mathrm{x}=1} \mathrm{ydy}
\end{aligned}
$$


The above support boundary conditions are also to be differentiated with respect to time $t$, and the condition (11) is to be adopted. Then we get:

$$
\begin{gathered}
\left.\frac{\partial^{4} \varphi}{\partial \mathrm{x}^{3} \partial \mathrm{y}}\right|_{\mathrm{x}=0}=-\left.\frac{\mathrm{k}_{1}}{\mathrm{EI}} \frac{\partial \varphi}{\partial \mathrm{y}}\right|_{\mathrm{x}=0} ;\left.\quad \frac{\partial^{4} \varphi}{\partial \mathrm{x}^{3} \partial \mathrm{y}}\right|_{\mathrm{x}=1}=\left.\frac{\mathrm{k}_{2}}{\mathrm{EI}} \frac{\partial \varphi}{\partial \mathrm{y}}\right|_{\mathrm{x}=1} \\
\left.\frac{\partial^{3} \varphi}{\partial \mathrm{x}^{2} \partial \mathrm{y}}\right|_{\mathrm{x}=0}=\left.\frac{\mathrm{C}_{1}}{\mathrm{EI}} \frac{\partial^{2} \varphi}{\partial \mathrm{x} \partial \mathrm{y}}\right|_{\mathrm{x}=0}+\left.\frac{\rho \mathrm{b}}{\mathrm{EI}} \int_{0}^{\mathrm{h}} \frac{\partial^{2} \varphi}{\partial \mathrm{t}^{2}}\right|_{\mathrm{x}=0} \mathrm{ydy} \\
\left.\frac{\partial^{3} \varphi}{\partial \mathrm{x}^{2} \partial \mathrm{y}}\right|_{\mathrm{x}=1}=\left.\frac{-\mathrm{C}_{2}}{\mathrm{EI}} \frac{\partial^{2} \varphi}{\partial \mathrm{x} \partial \mathrm{y}}\right|_{\mathrm{x}=1}+\left.\frac{\rho b}{\mathrm{EI}} \int_{0}^{\mathrm{h}} \frac{\partial^{2} \varphi}{\partial \mathrm{t}^{2}}\right|_{\mathrm{x}=1} \mathrm{ydy}
\end{gathered}
$$

Additionally we have:

$$
\left.\frac{\partial \varphi}{\partial y}\right|_{y=0}=\left.\frac{\partial \varphi}{\partial y}\right|_{y=h}
$$

The transverse vibration of a beam fully filled with liquid and supported at two points in an arbitrary way is completely defined by the differential equation (14) with the support conditions (16) and the Laplace equation (9) with the boundary conditions $(17) \div(19)$ for the liquid velocity:

$$
\begin{gathered}
\frac{\partial \varphi}{\partial z}=0 \text { for } z= \pm \frac{b}{2} \\
\frac{\partial \varphi}{\partial x}=-\left(y-\frac{h}{2}\right) \frac{\partial^{2} \varphi}{\partial x \partial y} \text { for } x=0 \text { and } x=1
\end{gathered}
$$

For free vibrations, taking into account (2) we arrive at the following form of equation (14):

$$
\begin{gathered}
\left.\frac{\partial^{5} \tilde{\varphi}}{\partial \mathrm{x}^{4} \partial \mathrm{y}}\right|_{\mathrm{y}=0}-\left.\mathrm{B} \frac{\partial^{3} \widetilde{\varphi}}{\partial \mathrm{x}^{2} \partial \mathrm{y}}\right|_{\mathrm{y}=0}= \\
=\omega^{2}\left[\left.\mathrm{~B}_{1} \frac{\partial \widetilde{\varphi}}{\partial \mathrm{y}}\right|_{\mathrm{y}=0}+\mathrm{B}_{3}\left(\left.\widetilde{\varphi}\right|_{\mathrm{y}=\mathrm{h}}-\left.\widetilde{\varphi}\right|_{\mathrm{y}=0}\right)\right]
\end{gathered}
$$

with the boundary conditions for the velocity potential $\tilde{\varphi}(\mathrm{x}, \mathrm{y}, \mathrm{z})$ :

$$
\begin{aligned}
& \left.\frac{\partial^{4} \tilde{\varphi}}{\partial \mathrm{x}^{3} \partial \mathrm{y}}\right|_{\mathrm{x}=0}=-\left.\frac{\mathrm{k}_{1}}{\mathrm{EI}} \frac{\partial \tilde{\varphi}}{\partial \mathrm{y}}\right|_{\mathrm{x}=0} ;\left.\frac{\partial^{4} \tilde{\varphi}}{\partial \mathrm{x}^{3} \partial \mathrm{y}}\right|_{\mathrm{x}=1}=\left.\frac{\mathrm{k}_{2}}{\mathrm{EI}} \frac{\partial \widetilde{\varphi}}{\partial \mathrm{y}}\right|_{\mathrm{x}=1} \\
& \left.\frac{\partial^{3} \tilde{\varphi}}{\partial \mathrm{x}^{2} \partial \mathrm{y}}\right|_{\mathrm{x}=0}=\left.\frac{\mathrm{C}_{1}}{\mathrm{EI}} \frac{\partial^{2} \tilde{\varphi}}{\partial \mathrm{x} \partial \mathrm{y}}\right|_{\mathrm{x}=0}-\left.\omega^{2} \mathrm{~B}_{3} \int_{0}^{\mathrm{h}} \tilde{\varphi}\right|_{\mathrm{x}=0} \mathrm{y} \mathrm{dy} \\
& \left.\frac{\partial^{3} \tilde{\varphi}}{\partial \mathrm{x}^{2} \partial \mathrm{y}}\right|_{\mathrm{x}=1}=\left.\frac{-\mathrm{C}_{2}}{\mathrm{EI}} \frac{\partial^{2} \tilde{\varphi}}{\partial \mathrm{x} \partial \mathrm{y}}\right|_{\mathrm{x}=1}-\left.\omega^{2} \mathrm{~B}_{3} \int_{0}^{\mathrm{h}} \tilde{\varphi}\right|_{\mathrm{x}=1} \mathrm{ydy}
\end{aligned}
$$

For the liquid inside the tank, the liquid velocity potential equation holds:

$$
\frac{\partial^{2} \tilde{\varphi}}{\partial \mathrm{x}^{2}}+\frac{\partial^{2} \widetilde{\varphi}}{\partial \mathrm{y}^{2}}=0
$$

with boundary conditions for the velocity:

$$
\left.\frac{\partial \tilde{\varphi}}{\partial y}\right|_{\mathrm{y}=0}=\left.\frac{\partial \widetilde{\varphi}}{\partial y}\right|_{\mathrm{y}=\mathrm{h}}
$$

$$
\begin{gathered}
\frac{\partial \tilde{\varphi}}{\partial z}=0 \text { for } z= \pm \frac{b}{2} \\
\frac{\partial \widetilde{\varphi}}{\partial x}=-\left(y-\frac{h}{2}\right) \frac{\partial^{2} \widetilde{\varphi}}{\partial x \partial y} \text { for } x=0 \text { and } x=1
\end{gathered}
$$

Let us introduce the „u” function defined as:

$$
\mathrm{u} \equiv \frac{\partial \widetilde{\varphi}}{\partial \mathrm{y}}
$$

Then the vibration equation (20) can be written as:

where:

$$
\left.\frac{d^{4} u}{d x^{4}}\right|_{y=0}-\left.B \frac{d^{2} u}{d x^{2}}\right|_{y=0}=K(x ; t)
$$

$$
\mathrm{K}(\mathrm{x} ; \mathrm{t}) \equiv \omega^{2}\left[\left.\mathrm{~B}_{1} \frac{\partial \tilde{\varphi}}{\partial \mathrm{y}}\right|_{\mathrm{y}=0}+\left.\mathrm{B}_{3} \widetilde{\varphi}\right|_{\mathrm{y}=0} ^{\mathrm{y}=\mathrm{h}}\right]
$$

Solving equation (27) is reduced to the search for the general integral of the homogeneous equation and a particular integral of the non-homogeneous equation. The general integral of the associate homogeneous equation is the following [13]:

$$
\mathrm{u}_{0}=\mathrm{D}_{1}+\mathrm{D}_{2} \mathrm{x}+\mathrm{D}_{3} \operatorname{shax}+\mathrm{D}_{4} \operatorname{chax}
$$

where:

$\mathrm{a} \equiv \sqrt{\mathrm{B}}$.

The particular integral of the non-homogeneous equation is searched for using the method of variation of constants. Finally, the integral of the non-homogeneous equation gets the form :

$$
\begin{gathered}
\mathrm{u}=\widetilde{\mathrm{D}}_{1}+\widetilde{\mathrm{D}}_{2} \mathrm{x}+\widetilde{\mathrm{D}}_{3} \operatorname{shax}+\widetilde{\mathrm{D}}_{4} \operatorname{chax}+ \\
+\frac{1}{\mathrm{a}^{2}}\left[\int_{0}^{\mathrm{x}} \xi \mathrm{K}(\xi, \mathrm{t}) \mathrm{d} \xi-\mathrm{x} \int_{0}^{\mathrm{x}} \mathrm{K}(\xi, \mathrm{t}) \mathrm{d} \xi\right]+ \\
+\frac{1}{\mathrm{a}^{3}}\left[\operatorname{shax} \int_{0}^{\mathrm{x}} \operatorname{cha} \xi \mathrm{K}(\xi, \mathrm{t}) \mathrm{d} \xi-\operatorname{chax} \int_{0}^{\mathrm{x}} \operatorname{sha} \xi \mathrm{K}(\xi, \mathrm{t}) \mathrm{d} \xi\right]
\end{gathered}
$$

Now, the boundary conditions (21) for the potential function will refer to the introduced function $\mathrm{u} \equiv \mathrm{u}(\mathrm{x}, \mathrm{t})$. These conditions will allow the constants $\operatorname{Di}(\mathrm{i}=1,2,3,4)$ to be determined. Then, these constants are to be put into the general integral (30), after which we get the solution in the form:

$$
\begin{gathered}
\left.\mathrm{u}(\mathrm{x}, 0 ; \mathrm{t}) \equiv \frac{\partial \widetilde{\varphi}}{\partial \mathrm{y}}\right|_{\mathrm{y}=0}=\mathrm{F}_{1}(\mathrm{x}) \int_{0}^{1} \xi \mathrm{Kd} \xi+\frac{1}{\mathrm{a}^{2}} \int_{0}^{\mathrm{x}} \xi \mathrm{Kd} \xi+ \\
+\mathrm{F}_{2}(\mathrm{x}) \int_{0}^{1} \mathrm{Kd} \xi-\frac{\mathrm{x}}{\mathrm{a}^{2}} \int_{0}^{\mathrm{x}} \mathrm{K} \mathrm{d} \xi+\left.\omega^{2} \mathrm{~F}_{3}(\mathrm{x}) \int_{0}^{\mathrm{h}} \widetilde{\varphi}\right|_{\mathrm{x}=1} \mathrm{ydy}+ \\
+\left.\mathrm{F}_{4}(\mathrm{x}) \omega^{2} \int_{0}^{\mathrm{h}} \tilde{\varphi}\right|_{\mathrm{x}=0} \mathrm{y} \mathrm{dy}+ \\
+\mathrm{F}_{5}(\mathrm{x}) \int_{0}^{1} \operatorname{sha} \xi \mathrm{Kd} \xi-\frac{\operatorname{chax}}{\mathrm{a}^{3}} \int_{0}^{\mathrm{x}} \mathrm{sha} \xi \mathrm{Kd} \xi+ \\
+\mathrm{F}_{6}(\mathrm{x}) \int_{0}^{1} \operatorname{cha} \xi \mathrm{Kd} \xi+\frac{\operatorname{shax}^{\mathrm{x}}}{\mathrm{a}^{3}} \int_{0}^{\mathrm{cha}} \operatorname{ch} \mathrm{Kd} \xi
\end{gathered}
$$




$$
\begin{gathered}
\mathrm{F}_{\mathrm{i}}(\mathrm{x}) \equiv \mathrm{u}_{\mathrm{i}}+\eta_{\mathrm{i}} \mathrm{x}+\alpha_{\mathrm{i}} \operatorname{shax}+\beta_{\mathrm{i}} \mathrm{chax} \\
\text { for } \mathrm{i}=1,2,3,5,6 \\
\text { and } \\
\mathrm{F}_{4}(\mathrm{x}) \equiv \mu_{4}+\left(\eta_{4}+\frac{\rho \mathrm{b}}{\mathrm{C}_{1}}\right) \mathrm{x}+\alpha_{4} \operatorname{shax}+\beta_{4} \operatorname{chax}
\end{gathered}
$$

The abovenamed coefficients: $u_{i}, \eta_{i}, \alpha_{i}, \beta_{i}$ (for $i=1,2,3,4,5,6$ ) are numeric constants calculated from the following formulas:

$$
\begin{aligned}
& \mu_{\mathrm{i}} \equiv-\beta_{\mathrm{i}}-\frac{\mathrm{a}^{3} \mathrm{EI}}{\mathrm{k}_{1}} \alpha_{\mathrm{i}} ; \eta_{\mathrm{i}} \equiv \frac{\mathrm{EIa}^{2}}{\mathrm{C}_{1}} \beta_{\mathrm{i}}-\mathrm{a} \alpha_{\mathrm{i}} \\
& \beta_{1} \equiv \frac{1}{\mathrm{Q}}\left[\frac{\mathrm{k}_{2}}{\mathrm{EI}} \operatorname{shal}+\frac{\mathrm{C}_{2} \mathrm{k}_{2}(\mathrm{chal}-1)}{\mathrm{a}(\mathrm{EI})^{2}}\right] \\
& \beta_{2} \equiv-\frac{1}{\mathrm{Q}}\left[\frac{\mathrm{k}_{2} \mathrm{l}}{\mathrm{EI}} \text { shal }+\frac{\mathrm{C}_{2} \mathrm{k}_{2} \mathrm{l} \cdot \mathrm{chal}}{\mathrm{a}(\mathrm{EI})^{2}}-\frac{\mathrm{C}_{2} \mathrm{k}_{2} \mathrm{l} \cdot \mathrm{shal}}{(\mathrm{a} \cdot \mathrm{EI})^{2}}+\frac{\mathrm{C}_{2} \mathrm{a}}{\mathrm{EI}}\left(\text { chal }+\frac{\mathrm{k}_{2}}{\mathrm{k}_{1}}\right)\right] \\
& \beta_{3} \equiv \frac{1}{\mathrm{Q}}\left[\frac{\rho \mathrm{ba}^{3}}{\mathrm{EI}}\left(\mathrm{chal}+\frac{\mathrm{k}_{2}}{\mathrm{k}_{1}}\right)+\frac{\rho \mathrm{bk}_{2}}{(\mathrm{EI})^{2}}(\mathrm{al}-\mathrm{shal})\right] \\
& \beta_{4} \equiv \frac{1}{\mathrm{Q}}\left[\frac{\mathrm{k}_{2} \mathrm{l} \rho \mathrm{pa}^{2} \mathrm{shal}}{\mathrm{C}_{1} \mathrm{EI}}+\frac{\mathrm{C}_{2} \rho \mathrm{\rho ba}^{3}}{\mathrm{C}_{1} \mathrm{EI}}\left(\text { chal }+\frac{\mathrm{k}_{2}}{\mathrm{k}_{1}}\right)+\frac{\mathrm{C}_{2} \mathrm{k}_{2} \rho \mathrm{b}}{\mathrm{C}_{1}(\mathrm{EI})^{2}}(\text { al chal }- \text { shal })\right]
\end{aligned}
$$

$$
\beta_{5} \equiv \frac{1}{\mathrm{Q}}\left[-\mathrm{a}^{2}+\frac{\mathrm{C}_{2} \mathrm{k}_{2}}{(\mathrm{aEI})^{2}}(\mathrm{chal}-1)-\frac{\mathrm{C}_{2} \mathrm{a} \cdot \mathrm{shal}}{\mathrm{EI}}-\frac{\mathrm{C}_{2} \mathrm{k}_{2} \mathrm{l} \cdot \mathrm{shal}}{(\mathrm{aEI})^{2}}-\frac{\mathrm{k}_{2} \text { lchal }}{\mathrm{EI}}-\frac{\mathrm{a}^{2} \mathrm{k}_{2} \text { chal }}{\mathrm{k}_{1}}-\frac{\mathrm{C}_{2} \mathrm{k}_{2} \text { ashal }}{\mathrm{k}_{1} \cdot \mathrm{EI}}\right]
$$$$
\beta_{6} \equiv \frac{1}{\mathrm{Q}}\left[\frac{\mathrm{C}_{2} \text { achal }}{\mathrm{EI}}-\frac{\mathrm{C}_{2} \mathrm{k}_{2} \text { shal }}{(\mathrm{aEI})^{2}}+\frac{\mathrm{k}_{2} 1 \text { shal }}{\mathrm{EI}}+\frac{\mathrm{k}_{2} \text { a shal }}{\mathrm{k}_{1}}+\frac{\mathrm{C}_{2} \mathrm{k}_{2} 1 \text { chal }}{\mathrm{a}(\mathrm{EI})^{2}}+\frac{\mathrm{C}_{2} \mathrm{k}_{2} \mathrm{a} \text { chal }}{\mathrm{k}_{1} \mathrm{EI}}\right]
$$

Here, $\mathrm{Q}$ is equal to:

$$
\begin{aligned}
& \mathrm{Q} \equiv \frac{\mathrm{C} \mathrm{k}_{2} \mathrm{a}}{(\mathrm{EI})^{2}}(2 \mathrm{chal}-\mathrm{a} 1 \mathrm{shal}-2)+\frac{\mathrm{C}_{2} \mathrm{a}^{4}}{(\mathrm{EI})}\left[\frac{\mathrm{k}_{2} 1}{\mathrm{aC}_{1}}(1-\text { chal })-\operatorname{shal}\left(1+\frac{\mathrm{k}_{2}}{\mathrm{k}_{1}}\right)\right]+ \\
& +\frac{\mathrm{k}_{2} \mathrm{a}^{2}}{\mathrm{EI}}\left[\operatorname{shal}\left(1+\frac{\mathrm{C}_{2}}{\mathrm{C}_{1}}\right)-\mathrm{al}\left(\operatorname{chal}+\frac{\mathrm{C}_{2}}{\mathrm{C}_{1}}\right)\right]-\mathrm{a}^{5}\left[\frac{\mathrm{k}_{2} 1}{\mathrm{aC}_{1}} \operatorname{shal}+\operatorname{chal}\left(\frac{\mathrm{k}_{2}}{\mathrm{k}_{1}}+\frac{\mathrm{C}_{2}}{\mathrm{C}_{1}}\right)+\frac{\mathrm{C}_{2} \mathrm{k}_{2}}{\mathrm{C}_{1} \mathrm{k}_{1}}+1\right] \\
& \alpha_{1} \equiv-\frac{1}{\mathrm{Q}}\left[\frac{\mathrm{k}_{2} \text { chal }}{\mathrm{EI}}+\frac{\mathrm{C}_{2} \mathrm{k}_{2}}{\mathrm{EIC}_{1}}+\frac{\mathrm{C}_{2} \mathrm{k}_{2} \text { shal }}{\mathrm{a}(\mathrm{EI})^{2}}\right] \\
& \alpha_{2} \equiv \frac{1}{\mathrm{Q}}\left[\frac{\mathrm{C}_{2} \mathrm{k}_{2}}{(\mathrm{aEI})^{2}}(1-\mathrm{chal})+\frac{\mathrm{C}_{2} \text { a shal }}{\mathrm{EI}}+\frac{\mathrm{k}_{2} 1 \mathrm{chal}}{\mathrm{EI}}+\frac{\mathrm{C}_{2} \mathrm{k}_{2} 1 \mathrm{shal}}{\mathrm{a}(\mathrm{EI})^{2}}\right] \\
& \alpha_{3} \equiv \frac{1}{\mathrm{Q}}\left[\frac{\rho \mathrm{bk}_{2}}{(\mathrm{EI})^{2}}(\mathrm{chal}-1)+\frac{\rho \mathrm{ba}^{2}}{\mathrm{EI}}\left(\frac{\mathrm{k}_{2} \mathrm{l}}{\mathrm{C}_{1}}-\mathrm{a} \text { shal }\right)\right] \\
& \alpha_{4} \equiv \frac{1}{\mathrm{Q}}\left[\frac{\mathrm{C}_{2} \mathrm{k \rho b}}{\mathrm{C}_{1}(\mathrm{EI})^{2}}(\mathrm{chal}-1-\mathrm{al} \text { shal })-\frac{\rho \mathrm{ba}^{2}}{\mathrm{C}_{1} \mathrm{EI}}\left(\mathrm{C}_{2} \text { a shal }+\mathrm{k}_{2} \text { l chal }\right)\right] \\
& \alpha_{5} \equiv \frac{1}{\mathrm{Q}}\left[\frac{\mathrm{k}_{2}}{\mathrm{EI}}\left(\frac{\mathrm{C}_{2} \text { shal }}{\mathrm{a}^{2} \mathrm{EI}}+\frac{\mathrm{chal}}{\mathrm{a}}\right)-\frac{\mathrm{k}_{2} \mathrm{l}}{\mathrm{C}_{1}}\left(\frac{\mathrm{C}_{2} \text { shal }}{\mathrm{EI}}+\frac{\mathrm{a} \text { chal }}{1}\right)+\frac{\mathrm{C}_{2}}{\mathrm{C}_{1}}\left(\frac{\mathrm{k}_{2} \text { chal }}{\mathrm{aEI}}-\mathrm{a}^{2} \text { shal }\right)\right] \\
& \alpha_{6} \equiv \frac{1}{\mathrm{Q}}\left[\frac{\mathrm{C}_{2} \mathrm{k}_{2}}{(\mathrm{aEI})^{2}}(1-\text { chal })-\frac{\mathrm{k}_{2} \text { shal }}{\mathrm{aEI}}+\mathrm{a}^{2}\left(1+\frac{\mathrm{C}_{2}}{\mathrm{C}_{1}} \text { chal }\right)+\frac{\mathrm{C}_{2} \mathrm{k}_{2}}{\mathrm{EIC}_{1}}\left(1 \text { chal }-\frac{\text { shal }}{\mathrm{a}}\right)+\frac{\mathrm{k}_{2} \text { al shal }}{\mathrm{C}_{1}}\right]
\end{aligned}
$$


The free vibration problem of the liquid-filled beam has been finally reduced to solving the Laplace equation (22) with boundary conditions $(23) \div(25)$ for the velocity potential and conditions (31) and (32), in which numeric coefficients are defined by formulas $(33) \div(37)$.

From the general boundary conditions for the velocity potential we can derive all particular cases referring to different cases of beam fixing at its both ends. An exception here is rigid fixing, for which the constants for axial and spiral springs (Fig. 1) k, C $\rightarrow \infty$.

\section{FREE VIBRATION OF A TANK COMPLETELY FILLED WITH LIQUID}

Let us return to equation (20), which is the free vibration equation for the liquid-filled beam. Neglecting the constant, the equation has the following form:

$$
\left.\frac{\partial^{5} \tilde{\varphi}}{\partial \mathrm{x}^{4} \partial \mathrm{y}}\right|_{\mathrm{y}=0}=\omega^{2}\left[\left.\mathrm{~A}_{1} \cdot \frac{\partial \widetilde{\varphi}}{\partial \mathrm{y}}\right|_{\mathrm{y}=0}+\mathrm{B}\left(\left.\tilde{\varphi}\right|_{\mathrm{y}=\mathrm{h}}-\left.\widetilde{\varphi}\right|_{\mathrm{y}=0}\right)\right]
$$

For the liquid inside the beam the Laplace equation (22) holds.

After integrating equation (38) four times with respect to the " $x$ " variable we get:

$$
\left.\frac{\partial \widetilde{\varphi}}{\partial y}\right|_{\mathrm{y}=0}=\omega^{2} \int_{0}^{1} \mathrm{~K}(\mathrm{x}, \xi) \mathrm{F}(\xi, 0) \mathrm{d} \xi
$$

where:

$$
\left.\mathrm{F}(\xi, 0) \equiv \mathrm{A}_{1} \cdot \frac{\partial \tilde{\varphi}}{\partial \mathrm{y}}\right|_{(\xi, 0)}+\mathrm{B}[\tilde{\varphi}(\xi, \mathrm{h})-\tilde{\varphi}(\xi, 0)]
$$

and $\mathrm{K}|\mathrm{x}, \xi|$ is a symmetrical kernel, i.e.

$$
\mathrm{K}|\mathrm{x}, \xi|=\mathrm{K}|\xi, \mathrm{x}|
$$

The kernel $\mathrm{K}|\mathrm{x}, \xi|$ defined in the two-dimensional domain, see Fig. 2, has the following polynomial form:

$$
K(x, \xi)=\left\{\begin{array}{l}
-\frac{\xi^{3}}{6}+\frac{x^{2}}{2}-\frac{x^{2} \xi^{2}}{1}+\frac{x^{2} \xi^{2}(x+\xi)}{21^{2}}-\frac{x^{3} \xi^{3}}{31^{3}} \\
\text { for } 0 \leq \xi \leq x \\
-\frac{x^{3}}{6}+\frac{\xi x^{2}}{2}-\frac{\xi^{2} x^{2}}{1}+\frac{\xi^{2} x^{2}(\xi+x)}{21^{2}}-\frac{\xi^{3} x^{3}}{31^{3}} \\
\text { for } x \leq \xi \leq 1
\end{array}\right.
$$

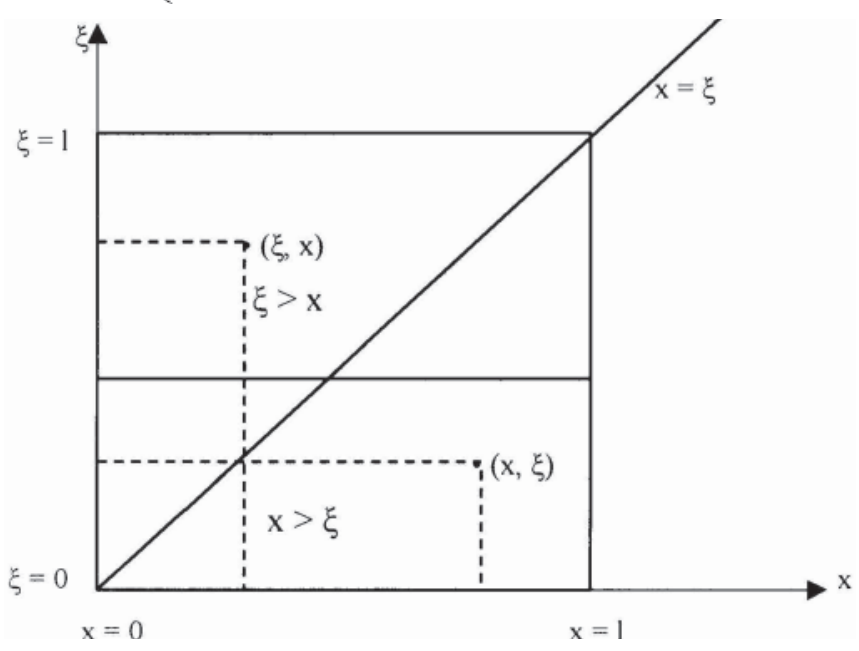

Fig. 2. Graphical representation of the symmetrical kernel
Finally, the free vibration problem for a beam fixed at both sides and filled with liquid within the linear range has been reduced to solving the Laplace equation in the two-dimensional domain D defined as (Fig. 3)

$$
\begin{gathered}
\mathrm{D} \equiv\left\{\begin{array}{l}
0 \leq \mathrm{x} \leq 1 \\
0 \leq \mathrm{y} \leq \mathrm{h}
\end{array}\right\} \\
\frac{\partial^{2} \widetilde{\varphi}}{\partial \mathrm{x}^{2}}+\frac{\partial^{2} \widetilde{\varphi}}{\partial \mathrm{y}^{2}}=0
\end{gathered}
$$

with differential conditions, and a differential-integral condition.

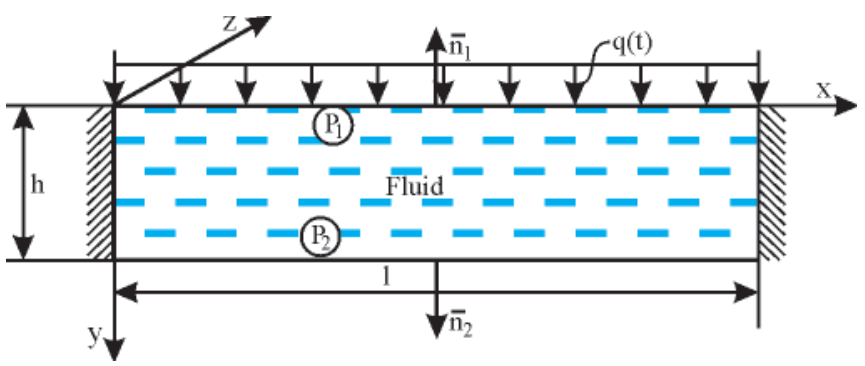

Fig. 3. Liquid-filled beam fixed at both sides

Let us assume that the liquid velocity potential function $\tilde{\varphi} \equiv \tilde{\varphi}(\mathrm{x}, \mathrm{y})$ is the product of two functions with separated variables in the form of the sum of an infinite series

$$
\widetilde{\varphi}(\mathrm{x}, \mathrm{y})=\sum_{\mathrm{n}=0}^{\infty} \cos \frac{\mathrm{n} \pi \mathrm{x}}{1} \mathrm{~g}_{\mathrm{n}}(\mathrm{y})
$$

The assumed potential has to fulfil the Laplace equation.

This way we arrive at the equation system determining free vibration frequencies and related modes. It is an infinite system of homogeneous algebraic equations. The problem of determining the free vibration frequency spectrum and modes is therefore reduced to the eigenvalue and eigenvector problem, written in the following matrix notation:

$$
\left[\mathrm{A} \omega^{2}-\mathrm{D}\right][\mathrm{CX}]=[0]
$$

where:

$A \equiv\left[a_{j n}\right]-$ symmetrical matrix of coefficients of kernel decomposition into the Fourier series, (formulas 46).

$$
\begin{gathered}
\mathrm{a}_{\mathrm{jn}}=\frac{1^{4}}{\left(\mathrm{jn} \pi^{2}\right)^{4}}\left[(\mathrm{j} \pi)^{4} \delta_{\mathrm{jn}}+24(\cos n \pi)(1-\cos \mathrm{j} \pi)\right] \\
\text { for } \mathrm{j}, \mathrm{n} \geq 1
\end{gathered}
$$

$$
\begin{gathered}
a_{00}=\frac{1^{4}}{720} \text { for } j, n=0 \\
a_{j 0}=\frac{1}{2} a_{\text {on }}=\frac{-1^{4}(1+\cos j \pi)}{2(j \pi)^{4}} \text { for } j \geq 1, n=0
\end{gathered}
$$

The matrices in the matrix equation (45) represent:

$\mathrm{D} \equiv\left[\mathrm{d}_{\mathrm{jj}}\right]-$ diagonal matrix, the element of which are calculated using the formula

$$
\begin{gathered}
d_{j j}=\left[A_{1}+\frac{2 l}{j \pi} B \text { th } \frac{j \pi h}{2 l}\right]^{-1} \text { for } j \geq 1 \\
d_{00}=\left[A_{1}+B h\right]^{-1} \text { for } j=0
\end{gathered}
$$

$\mathrm{CX} \equiv\left[\mathrm{C}_{\mathrm{j}} \mathrm{X}_{\mathrm{j}}\right]-$ single-column matrix, in which $\mathrm{C}_{\mathrm{j}}$ are unknown constants $\neq 0$ 
and $\mathrm{X}_{\mathrm{j}}$ are calculated using formulas (49) and (50):

$$
\mathrm{X}_{0} \equiv \mathrm{A}_{1}+\mathrm{Bh} \text { for } \mathrm{j}=0
$$

$$
X_{j} \equiv \frac{j \pi}{1}\left(e^{\frac{j \pi h}{1}}+1\right) A_{1}+2 B\left(e^{\frac{j \pi h}{1}}-1\right) \text { for } j \geq 1
$$

In a special case when the liquid is absent, i.e. when $\rho=0$, and $\mathrm{B}=0$ the elements of the abovenamed matrices are determined using the following relations:

$$
\mathrm{d}_{\mathrm{ij}}=\frac{1}{\mathrm{~A}_{1}} \text { for } \mathrm{j} \geq 0
$$

and

$$
\begin{gathered}
X_{0}=A_{1} \text { for } j=0 \\
X_{j}=\frac{j \pi}{1}\left(e^{\frac{j \pi h}{1}}+1\right) A_{1} \text { for } j \geq 1
\end{gathered}
$$

In this case the matrix equation (45) describes free vibrations of the system without liquid.

Behaviour of the liquid inside the beam during free vibrations.

Having known the liquid velocity potential we can determine the velocity field for the liquid:

$$
\bar{V}=\frac{\partial \widetilde{\varphi}}{\partial x} \bar{i}+\frac{\partial \widetilde{\varphi}}{\partial y} \bar{j}
$$

where:

$\bar{i}, \bar{j}-$ unit vectors of the Cartesian coordinate system 0xy

and

$$
\begin{gathered}
\frac{\partial \widetilde{\varphi}}{\partial x}=-\sum_{n=1}^{\infty} C_{n} \frac{n \pi}{1} \sin \frac{n \pi x}{1}\left[e^{\frac{n \pi}{1} y}-e^{\frac{n \pi}{1}(h-y)}\right] \\
\frac{\partial \widetilde{\varphi}}{\partial y}=C_{0}+\sum_{n=1}^{\infty} C_{n} \frac{n \pi}{1} \cos \frac{n \pi x}{1}\left[e^{\frac{n \pi}{1} y}+e^{\frac{n \pi}{1}(h-y)}\right]
\end{gathered}
$$

Having determined components of the liquid velocity field we can find the equation for the family of streamlines as:

$\psi(\mathrm{x}, \mathrm{y})=-\mathrm{C}_{0} \mathrm{x}-\sum_{\mathrm{n}=1}^{\infty} \mathrm{C}_{\mathrm{n}} \sin \frac{\mathrm{n} \pi \mathrm{x}}{\mathrm{l}}\left[\mathrm{e}^{\frac{\mathrm{n} \pi}{1} \mathrm{y}}+\mathrm{e}^{\frac{\mathrm{n} \pi}{1}(\mathrm{~h}-\mathrm{y})}\right]$

From the condition $\psi=$ const we obtain the family of streamlines.

The pressure is calculated from the following Bernoulli relation:

$$
p(x, y ; t)=-\rho\left(\frac{\partial \varphi}{\partial t}\right)
$$

or taking into account the velocity term [12]:

$$
p(x, y ; t)=-\rho\left(\frac{\partial \varphi}{\partial t}+\frac{1}{2} \operatorname{grad}^{2} \varphi\right)
$$

Taking only into account the amplitude of the dynamic pressure we arrive at:

$$
\begin{gathered}
\tilde{p}(x, y)=\rho \omega\left\{C_{0}\left(y-\frac{h}{2}\right)+\right. \\
\left.+\sum_{n=1}^{\infty} C_{n} \cos \frac{n \pi x}{1} \cdot\left[e^{\frac{n \pi}{1} y}-e^{\frac{n \pi}{1}(h-y)}\right]\right\}
\end{gathered}
$$

\section{FREE VIBRATION OF A BEAM PARTIALLY FILLED WITH LIQUID}

A case which most often happens in practice is when the tank is partially filled with liquid. A thin-wall prismatic beam of dimensions $1 \times b \times h$ is assumed, with the liquid of density $\rho$ inside it. The height to which the beam is filled with the liquid is smaller that the $\mathrm{h}$ dimension of the beam.

For the 0xyz system assumed as in Fig. 4, the free surface of the liquid has a wavy shape described by the equation $\mathrm{y}=\mathrm{Y} / \mathrm{x}, \mathrm{z}, \mathrm{t} /$.

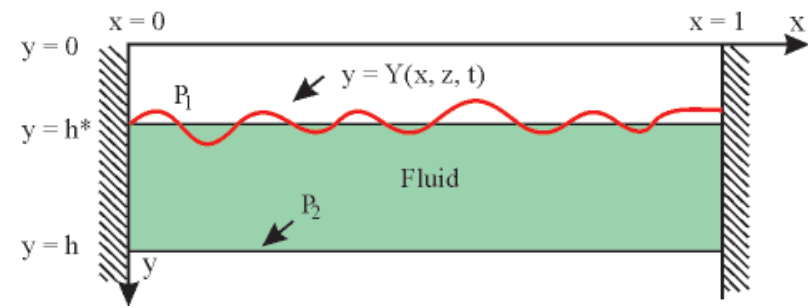

Fig. 4. Beam partially filled with liquid

Like in the previous case, we have to adopt model assumptions for the beam and the liquid. The liquid velocity potential $\varphi / \mathrm{x}, \mathrm{y}, \mathrm{z}, \mathrm{t} /$ fulfils the Laplace equation:

$$
\nabla^{2} \varphi=0
$$

as, by default, the flow of incompressible liquid in the beam is irrotational within the entire domain $\mathrm{D}$ defined as:

$$
D \equiv\left\{\begin{array}{l}
0 \leq x \leq 1 \\
-\frac{b}{2} \leq z \leq \frac{b}{2} \\
Y / x, z / \leq y \leq h
\end{array}\right\}
$$

Assuming two-dimensional waves, we introduce a constant average height of filling $\mathrm{H} \equiv \mathrm{h}-\mathrm{h}^{*}$. After further transformations of equation (45) we arrive at infinite homogeneous equation system, which can be written in the matrix form:

where:

$$
\left[\mathrm{A} \omega^{2}\right][\mathrm{CX}]=[0]
$$

$\mathrm{A} \equiv\left[\mathrm{a}_{\mathrm{jn}}\right] \quad-$ symmetrical matrix of coefficients of kernel expansion into the Fourier series, the elements of which are calculated using formulas (38)

$\mathrm{CX} \equiv\left[\mathrm{C}_{\mathrm{j}} \mathrm{X}_{\mathrm{j}}\right]-$ single-column matrix, in which $\mathrm{C}_{\mathrm{j}}$ are constant unknowns $\neq 0$; and $X_{\mathrm{j}}$ are calculated in the following way:

$$
\begin{gathered}
X_{j}=A_{1} s\left[\left(g s+\omega^{2}\right) e^{s\left(h-h^{*}\right)}-\left(g s-\omega^{2}\right) e^{s\left(h^{*}-h\right)}\right]+ \\
+B\left[\left(g s+\omega^{2}\right) e^{s\left(h-h^{*}\right)}+\left(g s-\omega^{2}\right) e^{s\left(h^{*}-h\right)}-2 g s\right] \\
\text { for } \mathrm{j} \geq 1
\end{gathered}
$$

and

$$
\mathrm{X}_{0}=\mathrm{A}_{1}+\mathrm{B}\left(\mathrm{h}-\mathrm{h}^{*}\right)
$$

$\mathrm{D} \equiv\left[\mathrm{d}_{\mathrm{jj}}\right] \quad-$ diagonal matrix, the elements of which are determined using the following formulas:

$$
\begin{gathered}
\text { for } \mathrm{j}=0 \quad \mathrm{~d}_{00}=\frac{1}{\mathrm{~A}_{1}+\mathrm{B}\left(\mathrm{h}-\mathrm{h}^{*}\right)} \\
\mathrm{d}_{\mathrm{jj}}=\left[\mathrm{A}_{1}+\frac{\mathrm{B}}{\mathrm{s}} \frac{\operatorname{gsth} \frac{\mathrm{s}}{2}\left(\mathrm{~h}-\mathrm{h}^{*}\right)+\omega^{2}}{\mathrm{gs}+\omega^{2} \operatorname{cth} \mathrm{s}\left(\mathrm{h}-\mathrm{h}^{*}\right)}\right]^{-1} \text { for } \mathrm{j} \geq 1 \\
\mathrm{~s}=\frac{\mathrm{j} \pi}{\mathrm{l}}
\end{gathered}
$$


Here we can make distinction between two limiting cases of liquid filling, which are :

I. where $\mathrm{h}^{*} \rightarrow \mathrm{h}-$ liquid is absent

II. where $\mathrm{h}^{*} \rightarrow 0 \quad$ - full filling.

Case I:

$\rho=0$ i.e. $B=0$ and then:

$$
\begin{gathered}
X_{0}=A_{1} ; X_{j}=\frac{2 j \pi}{1} A_{1} \omega^{2} \text { for } j \geq 1 \\
d_{00}=d_{j j}=\frac{1}{A_{1}}
\end{gathered}
$$

Case II:

$\rho \neq 0 ; \mathrm{h}-\mathrm{h}^{*} \rightarrow \mathrm{h}$, then:

$$
\left.\begin{array}{r}
X_{j}=A_{1} s\left[\left(g s+\omega^{2}\right) e^{s h}-\left(g s-\omega^{2}\right) e^{-s h}\right]+ \\
+B\left[\left(g s+\omega^{2}\right) e^{s h}+\left(g s-\omega^{2}\right) e^{-s h}-2 g s\right] \\
d_{00}=\frac{1}{A_{1}+B h} \quad \text { for } j=0 \\
d_{j j}=\left[A_{1}+\frac{B\left(g s t h\left(\frac{s h}{2}\right)+\omega^{2}\right)}{s\left(g s+\omega^{2} \operatorname{cth}(s h)\right)}\right]^{-1} \text { for } j \geq 1
\end{array}\right\}
$$

In all above formulas $\mathrm{A}_{1}$ and $\mathrm{B}$ are the constants equal, respectively, to:

$$
A_{1} \equiv \frac{\rho_{b} A}{E I} \quad ; \quad B \equiv \frac{\rho b}{E I}
$$

Case II is not included in the earlier obtained relations, as there was no free surface in those cases.

\section{NUMERICAL SOLUTIONS}

The equation systems (45) and (62) were solved numerically using an in-home code written on PC computer. An infinite system of algebraic equations was obtained, which is fully regular. According to a theorem, a method of successive sections can be applied in this case. For the assumed section dimension " $n$ " the code creates system matrices and solves the eigenvalue and eigenvector problem. The code calculates and prints the liquid pressure and velocity fields at a selected point of the area occupied by the liquid. For partial filling, the code additionally prints the deflection and the shape of the free surface. For this case dynamic pressure amplitudes are also calculated. Along with hydrodynamic frequencies in the deformable tank, the frequencies of the same volume of liquid in a rigid tank are determined as well.

\section{CONCLUSIONS FROM THE OBTAINED SOLUTIONS}

For four height-to-length ratios, i.e.

$$
\mathrm{h} / 1 \in\{0.02,0.1,0.5,1\}
$$

transverse vibration frequency spectra were obtained, making use of the here presented theory and a simplified formula, for the tank filled with liquid.

Figs $(5) \div(6)$ show the frequency spectra for six first frequencies and that for the tank without liquid (curves marked $\rho=0$ in the figures).

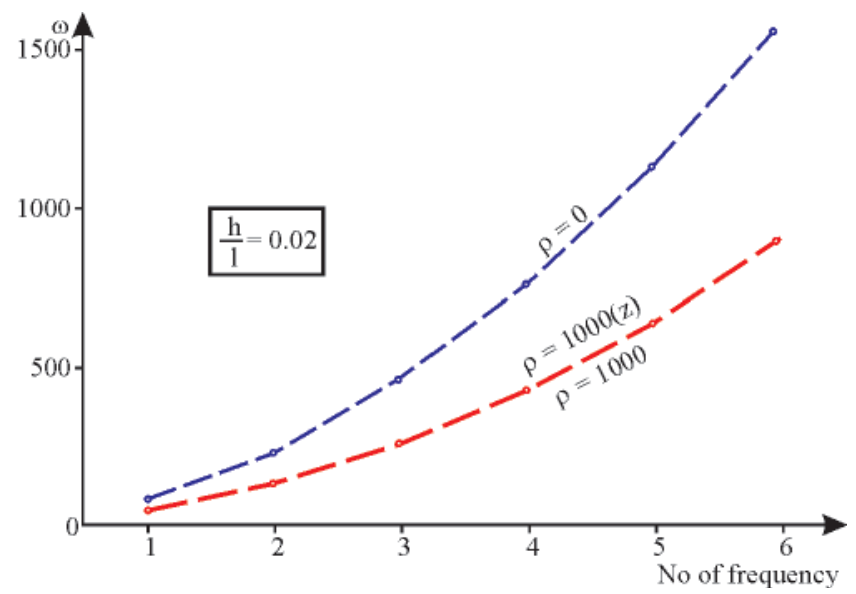

Fig. 5. Vibration frequency spectrum for the tank fully filled with liquid: $h / l=0.02$

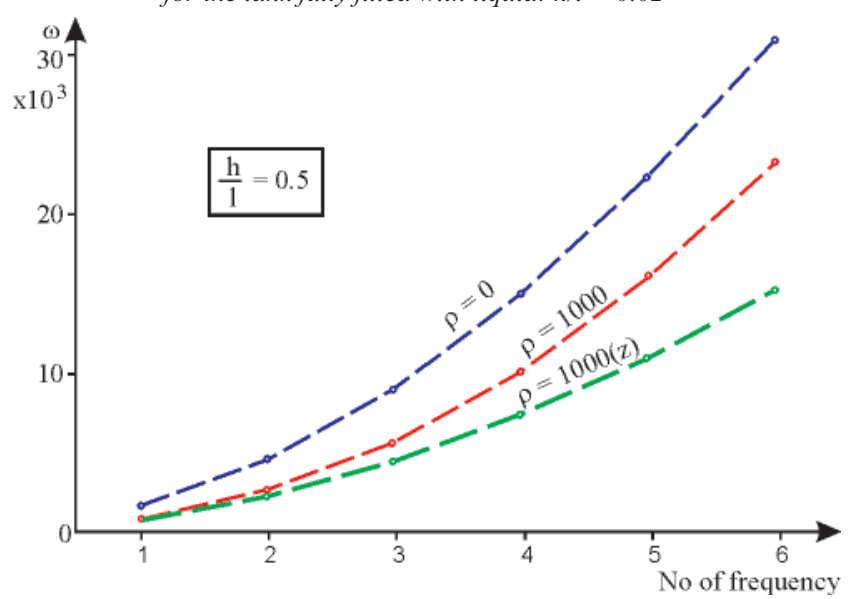

Fig. 6. Vibration frequency spectrum for the tank fully filled with liquid: $h / l=0.5$

Dynamic pressure amplitudes and velocity field components were determined at all nodes of the grid of the dimensions $\mathrm{l} / 40 \times \mathrm{h} / 10$.

This made the basis for preparing diagrams of dynamic pressure distributions on tank walls for each of six first modes and for three $\mathrm{h} / \mathrm{l}$ ratios. The pressure diagrams refer to the half-length of the tank and the fixed top wall. Sample cases are shown in Figs. $(7 \div 8)$.

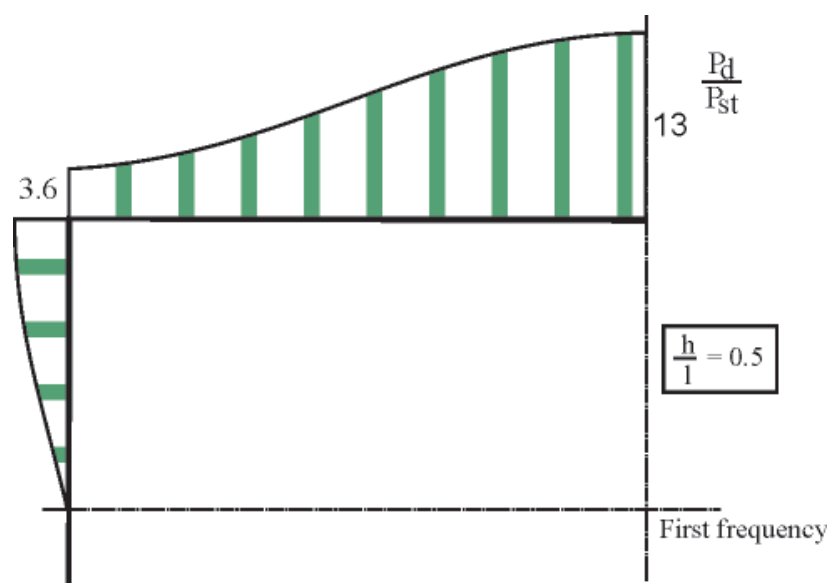

Fig. 7. Pressure distribution on tank walls for the first frequency and $h / l=0.5$

In the case of the tank partially filled with liquid, frequency spectra are shown for each of the above named $\mathrm{h} / \mathrm{l}$ ratios and four cases of liquid filling, i.e.

$\mathrm{H} / \mathrm{h} \in\{0.25,0.5,0.75,1\}$ 


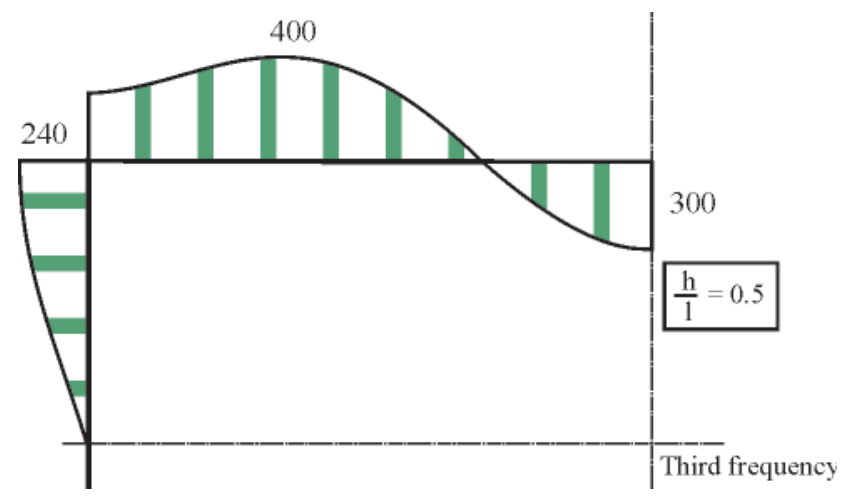

Fig. 8. Pressure distribution on tank walls

where: for the third frequency and $h / l=0,5$

$\mathrm{H}-$ liquid column height.

Additionally, sample diagrams shown in Figs. $(9 \div 10)$ present, as a comparison, frequency spectra which neglect the dynamic condition on the free surface of the liquid (curves marked "I").

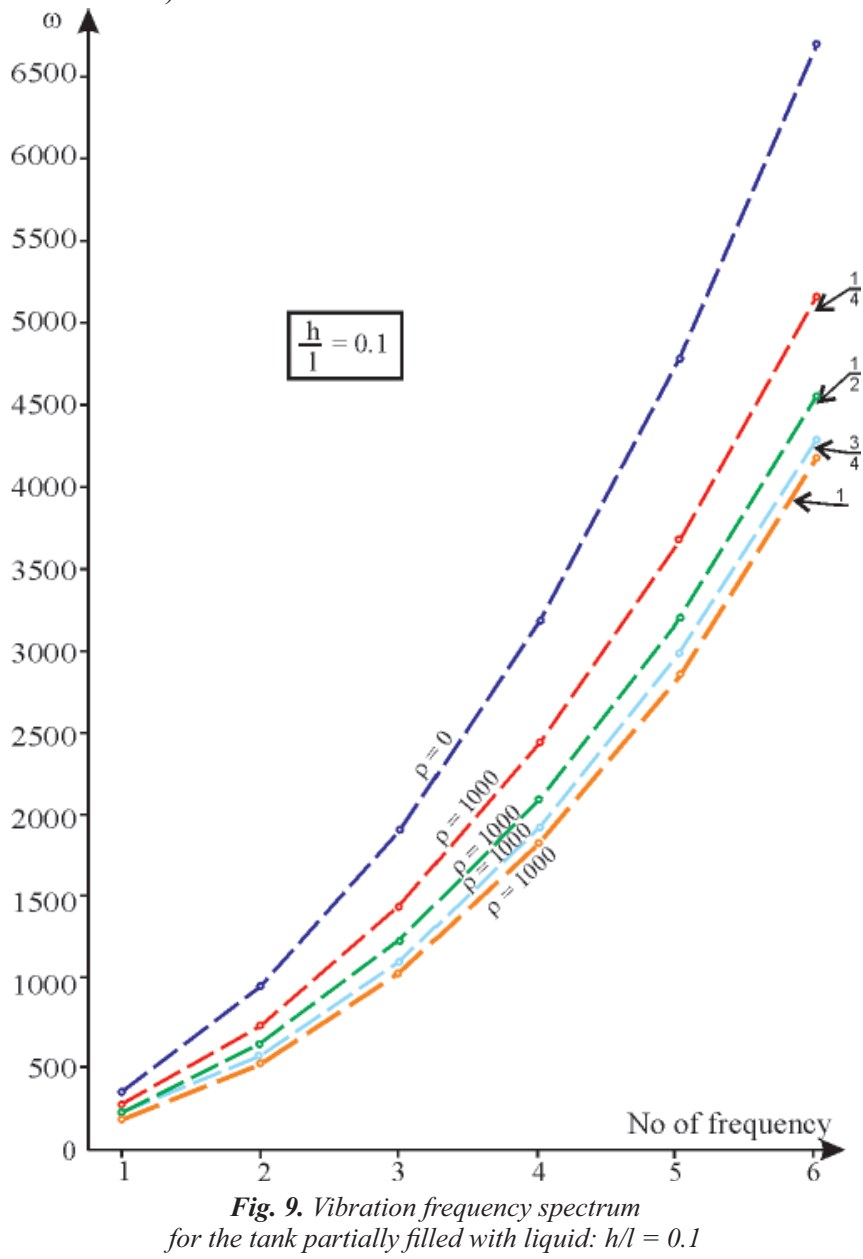

Figures (11) show beam frequency diagrams for the first frequencies. The diagrams present the beam frequencies according to the present theory (marked "B"), those calculated as the reduced frequencies (marked " $z$ "), the hydrodynamic or "liquid" frequencies (marked "c") and the frequencies of the liquid itself in a rigid tank (marked "w").

Then, for two frequencies and four h/l ratios, Figs. $(12 \div 13)$ show dynamic pressure distributions for the bottom and top walls, for all four fillings. These diagrams refer to the half-length of the tank. Obviously, the point symmetry is observed for even frequencies and the axial symmetry for odd frequencies.

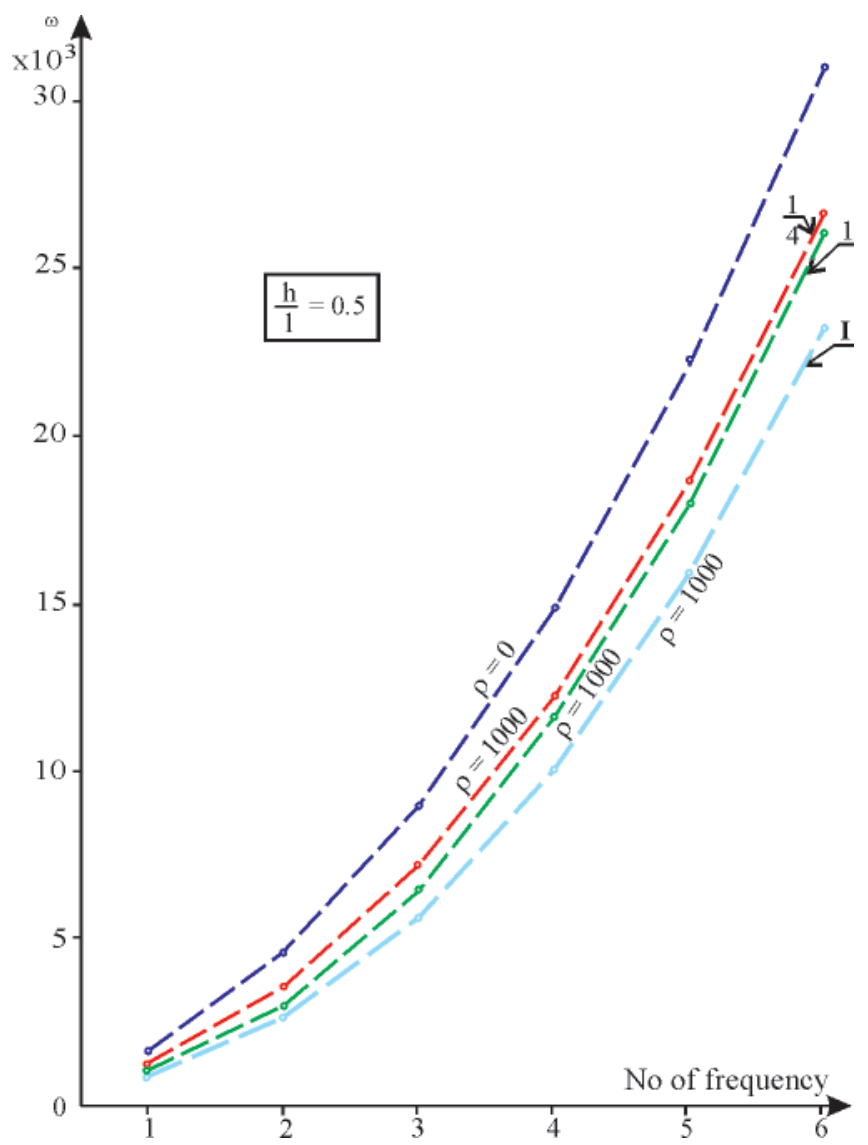

Fig. 10. Vibration frequency spectrum for the tank partially filled with liquid: $h / l=0.5$

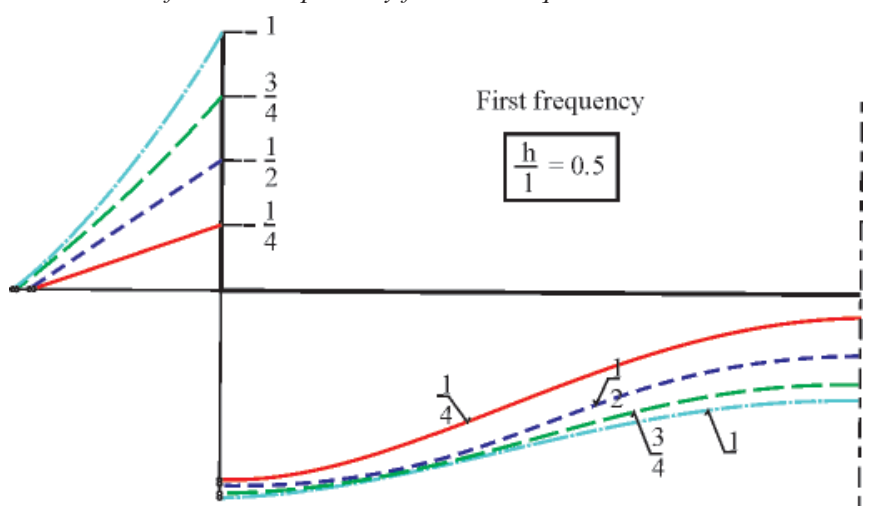

Fig. 12. Dynamic pressure distribution for tank bottom and top walls: $h / l=0.5$, the first frequency

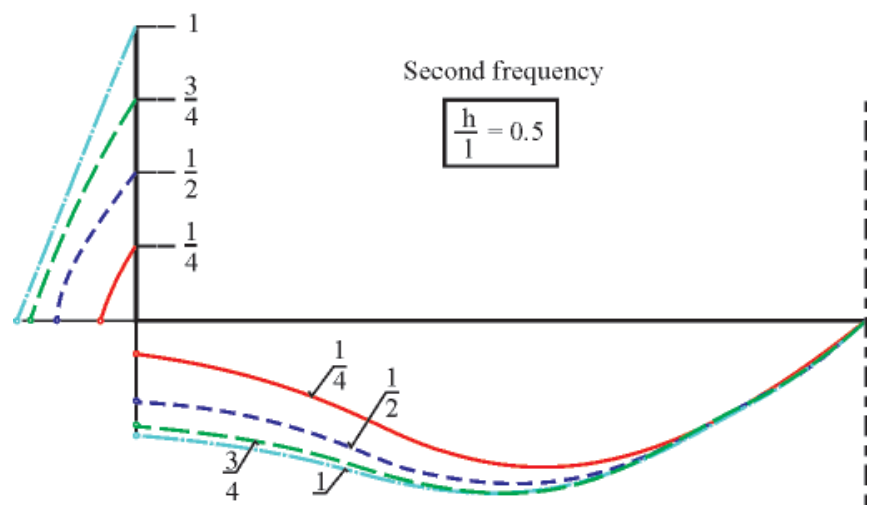

Fig. 13. Dynamic pressure distribution

for tank bottom and top walls: $h / l=0.5$, the second frequency

The next figures, Figs. $(14 \div 15)$, show tank bottom deflection amplitudes and the shape of the liquid free surface for the first three frequencies, and for different $\mathrm{h} / \mathrm{l}$ ratios and tank fillings. 


\section{First frequency}
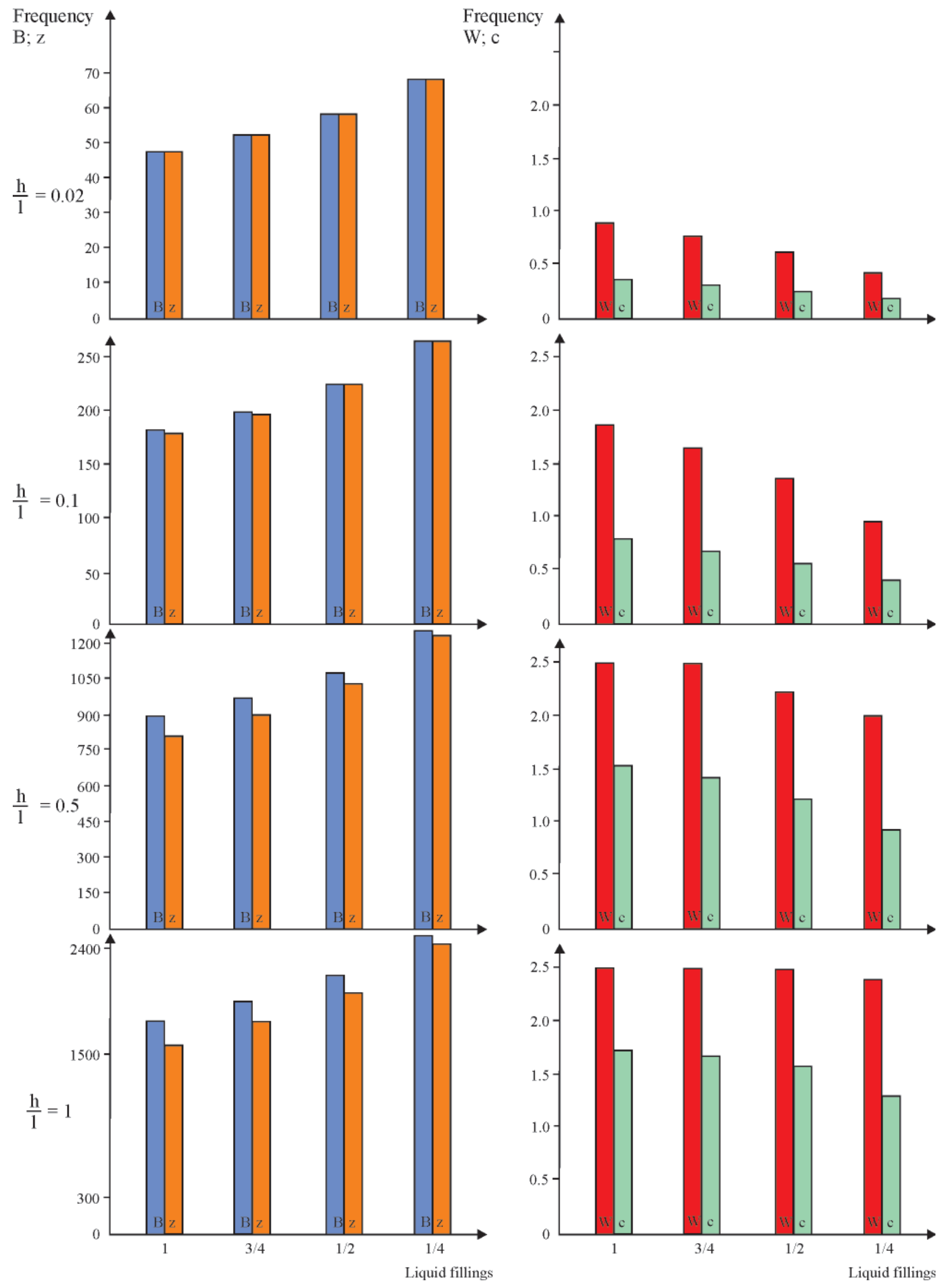


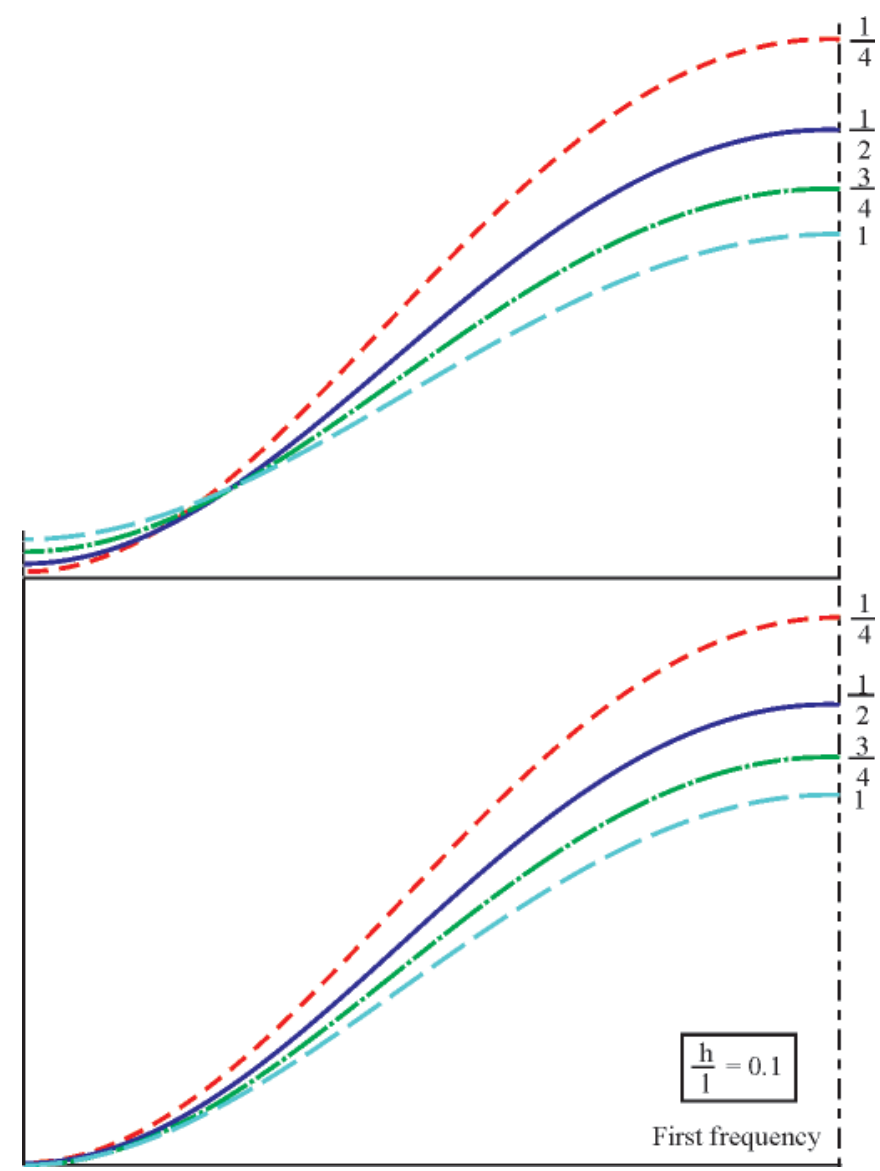

Fig. 14. Tank bottom deflection amplitudes and free surface shapes: $h / l=0.1$; the first frequency

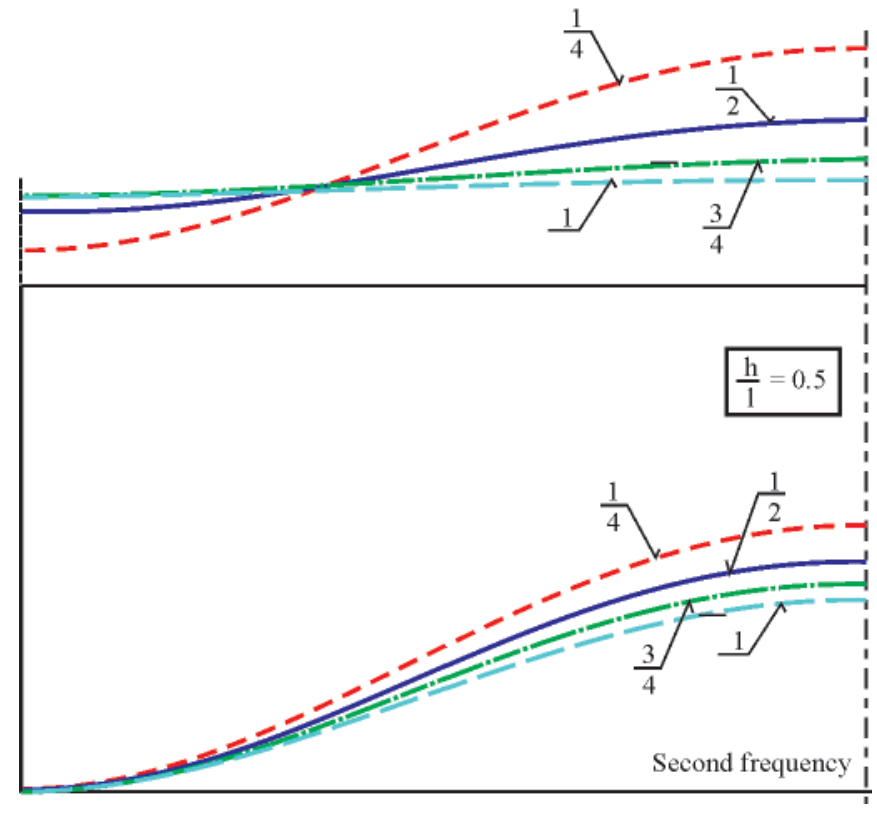

Fig. 15. Tank bottom deflection amplitudes and free surface shapes: $h / l=0.5$; the second frequency

\section{CONCLUSIONS}

The presence of the liquid in the beam decreases vibration frequencies, compared to the frequency spectrum of the beam without liquid.

The spectrum levels determined based on the here presented theory depend on the $\mathrm{h} / \mathrm{l}$ ratio, and are above those calculated using a simplified formula.
Reducing the volume of the liquid in the beam leads to the reduction in percentage differences between the abovementioned spectra, and in the limiting case of the absence of the liquid the frequency spectra are identical with each other.

O Taking into account the dynamic condition on the free surface moves the spectrum towards higher values. For low beams $\mathrm{h} / \mathrm{l}<0.1$ this effects is negligible.

When $\mathrm{h} / \mathrm{l}$ increases, the flow of the liquid in the vicinity of the tank walls becomes more intensified. Vertical velocities of the liquid particles in the central layer and on the surface of the moistened tank bottom differ significantly, starting from the second and higher frequencies. For beams fully filled with liquid and the ratio $\mathrm{h} / \mathrm{l}=1$ the motion of the liquid reflects the membrane principle.

O Horizontal velocity components of the liquid particles move the hydrodynamic pressure distribution towards tank tops, which increases the loads of the top walls with increasing $\mathrm{h} / \mathrm{l}$.

\section{BIBLIOGRAPHY}

1. Cho J.R., Lee H.W. and Kim K.W.: Free vibration analysis of baffled liquid-storage tanks by the structural-acoustic finite element formulation, J.Sound and Vibration, 258, 847, 2002

2. Kekana M. and Badur J.: Modeling of beams using the reduced integration technique-statics and free vibration, Res.Dev.J., 16, 9,2000

3. Kobayashi E., Asai S.: A study on a mathematical model for maneuvering motions at low speed (in Japanese), J Kansai Soc. Nav. Archit., 193, 27-37, 1984

4. Lamb H.: Hydrodynamics, Cambridge University Press, 1975

5. Liu W. K., Ma D. C.: Computer implementation aspects for fluid-structure interaction problems, Comp. Meth. in Applied Mech. and Eng., 31, 2, 129-148, 1982

6. Morand H. J. P., Ohayon R.: Fluid Structure Interaction, J. Wiley \& Sons, Chichester, 1995

7. Parszewski Z.: Drgania i dynamika maszyn, WNT Warszawa, 1982

8. Sawicki A.: Mechanika Kontinuum - Wprowadzenie, IBW PAN, Gdańsk, 1994

9. Trębacki K.: Drgania swobodne cieczy w sztywnych i odkształconych zbiornikach wypełnionych ciecza, Pr. IO nr 2268/MR-1141/85, Gdańsk, 1985

10.Trębacki K.: Algorytm obliczania drgań swobodnych zbiornika wypetnionego ciecza, PB IO nr 223/CPBR 9.5-231/87, Gdańsk, 1987

11.Trębacki K.: Drgania swobodne zbiorników wypetnionych ciecza. Cz. I-Analiza teoretyczna, Zeszyty Naukowe Politechniki Gdańskiej nr 418, Budownictwo Okrętowe XLVII, ss. $197-210,1990$

12.Trębacki K.: Wpływ bezwładności obrotowej ścian szczytowych zbiornika z ciecza na widmo czestości drgań, Polish Academy of Sciences, Branch in Gdańsk, Marine Technology Transaction Vol. 4, pp. 224-246, 1993

13.Trębacki K.: Hydrodynamika zbiornika wypetnionego częściowo ciecza, XXI Sesja Naukowa Okrętowców, Gdańsk, 2004

14.Zienkiewicz O.C. and Taylor R.L.: The Finite Element Metod, McGraw-Hill, NY, 1991.

\section{CONTACT WITH THE AUTHOR}

Kazimierz Trębacki, Ph. D.

Faculty of Ocean Engineering and Ship Technology

Gdansk University of Technology Narutowicza 11/12

80-952 Gdansk, POLAND

e-mail : katre@pg.gda.pl 